\title{
Prengel, Annedore
}

\section{Egalitäre Differenz in der Bildung}

formal überarbeitete Version der Originalveröffentlichung in: formally revised edition of the original source in:

Lutz, Helma [Hrsg.]; Wenning, Norbert [Hrsg.]: Unterschiedlich verschieden. Differenz in der Erziehungswissenschaft. Opladen : Leske + Budrich 2001, S. 93-107

Bitte verwenden Sie in der Quellenangabe folgende URN oder DOI /

Please use the following URN or DOI for reference:

urn:nbn:de:0111-opus-26218

$10.25656 / 01: 2621$

https://nbn-resolving.org/urn:nbn:de:0111-opus-26218

https://doi.org/10.25656/01:2621

\section{Nutzungsbedingungen}

Gewährt wird ein nicht exklusives, nicht übertragbares, persönliches und beschränktes Recht auf Nutzung dieses Dokuments. Dieses Dokument ist ausschließlich für den persönlichen, nicht-kommerziellen Gebrauch bestimmt. Die Nutzung stellt keine Übertragung des Eigentumsrechts an diesem Dokument dar und gilt vorbehaltlich der folgenden Einschränkungen: Auf sämtlichen Kopien dieses Dokuments müssen alle Urheberrechtshinweise und sonstigen Hinweise auf gesetzlichen Schutz beibehalten werden. Sie dürfen dieses Dokument nicht in irgendeiner Weise abändern, noch dürfen Sie dieses Dokument für öffentliche oder kommerzielle Zwecke vervielfältigen, öffentlich ausstellen, aufführen, vertreiben oder anderweitig nutzen.

Mit der Verwendung dieses Dokuments erkennen Sie die Nutzungsbedingungen an.

\section{Terms of use}

We grant a non-exclusive, non-transferable, individual and limited right to using this document

This document is solely intended for your personal, non-commercial use. Use of this document does not include any transfer of property rights and it is conditional to the following limitations: All of the copies of this documents must retain all copyright information and other information regarding legal protection. You are not allowed to alter this document in any way, to copy it for public or commercial purposes, to exhibit the document in public, to perform, distribute or otherwise use the document in public.

By using this particular document, you accept the above-stated conditions of use.

\section{Kontakt / Contact:}

\section{peDOCS}

DIPF | Leibniz-Institut für Bildungsforschung und Bildungsinformation Informationszentrum (IZ) Bildung

E-Mail: pedocs@dipf.de

Internet: www.pedocs.de

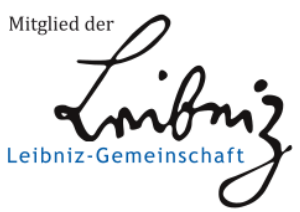


Helma Lutz/Norbert Wenning (Hrsg.)

\section{Unterschiedlich verschieden \\ Differenz in der Erziehungswissenschaft}

Leske + Budrich, Opladen 2001 
Die erste Auflage dieses Band erschien 2001 im Verlag Leske + Budrich, Opladen (jetzt VS-Verlag, Wiesbaden) und hatte folgende ISBN:

ISBN 3-8100-2854-1

Diese Version des Textes wird von der Herausgeberin und dem Herausgeber verantwortet. Sie macht den Gesamttext mit allen Beiträgen des genannten Bandes durch eine Open-Access-Publikation frei zugänglich. Das Werk ist dennoch insgesamt und in seinen einzelnen Teilen urheberrechtlich geschützt. Das Gesamtwerk bzw. die Einzelbeiträge dürfen nur mit Zustimmung der jeweiligen Autorinnen bzw. Autoren vervielfältigt, übersetzt oder in anderer Weise außerhalb der Grenzen des Urheberrechts genutzt werden. Jede kommerzielle Nutzung ist ausgeschlossen. 


\section{Inhaltsverzeichnis}

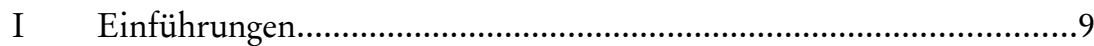

Differenzen über Differenz - Einführung in die Debatten.........................11

Helma Lutz, Norbert Wenning

Die Verwandlung der Philosophie in eine historische Diagnostik

der Differenzen.......................................................................................25

Rita Casale

Differenz und Differenzierung in soziologischer Perspektive

Frank Hillebrandt

Aspekte der angloamerikanischen pädagogischen Differenzdebatte:

Überlegungen zur Kontextualisierung.

Karin Amos

Egalitäre Differenz in der Bildung.

Annedore Prengel

II Disziplintheoretische Zugänge zu Differenz

Feministische Perspektiven auf „Differenz“ in Erziehungsund Bildungsprozessen.

Martina Löw

Das Soziale und die Differenz. Zur (De-)Thematisierung von

Differenz in der Sozialpädagogik.

Susanne Maurer 
Die Rezeption von Differenzdiskussionen in der Vergleichenden Erziehungswissenschaft

Karin Amos

Differenz als Konstitutionsproblem der Sonderpädagogik

Günther Opp, Michael Fingerle, Kirsten Pubr

III Kategorien zur Konstruktion von Differenz. .177

Frauen/Männer, Kinder/Erwachsene.

Rolf Nemitz

Kultur als Differenzierungskategorie.

Thomas Höhne

Differenz als Rechenaufgabe: über die Relevanz der Kategorien

Race, Class und Gender.....

Helma Lutz

An-, Zu- und Ungehörigkeiten Jugendlicher: Herkunft als Auskunft?....231 Clemens Dannenbeck, Hans Lösch, Felicitas Eßer

IV Zur Produktion von Differenz

Dichotome Differenzen und antirassistische Praxis. .251

Rudolf Leiprecht, Susanne Lang

Differenz durch Normalisierung. .275

Norbert Wenning

Die Autorinnen und Autoren. 


\section{Danksagung}

Dieses Buch ist nur zustande gekommen durch die Mitarbeit vieler HelferInnen mit unterschiedlichsten Beiträgen.

Wir bedanken uns insbesondere bei Ingrid Gogolin, Marianne KrügerPotratz, Rudolf Leiprecht, Karl-Ernst Ackermann und den Studierenden des Seminars „Differenzdebatten in der Erziehungswissenschaft“, das im Wintersemester 1999/2000 an der Westfälischen Wilhelms-Universität Münster stattfand, für interessante Hinweise und Kommentare zur inhaltlichen Gestaltung des Buches. Die Autorinnen und Autoren hatten sich mit vielfachen Änderungswünschen unsererseits zu befassen und dabei Ausdauer zu zeigen. Ulrike Fromm, Jeannette Stiller und Bernhard Rosenkötter haben mit viel Geduld die Korrekturen der diversen Versionen des Buches vorgenommen und umgesetzt. Dafür ein besonderes Dankeschön.

Wir hoffen, dass dieses Buch seinen Leserinnen und Lesern Anlass gibt zu heftigen Diskussionen, zu Revisionen und Perspektivwechseln, vielleicht auch zu Widerspruch.

Münster, Sommer 2000

Helma Lutz und Norbert Wenning

Hinweise zur Wiederauflage:

Die erneute Auflage dieses Bandes erfolgt, weil die Verlagsfassung im Buchhandel vergriffen ist und zugleich wiederholt Nachfragen nach dem Text an uns gerichtet werden. Eine vollständige Überarbeitung der Beiträge wäre nach fast zehn Jahren reizvoll, erscheint uns aber als recht aufwändig. Zudem hat sich die Debatte um Differenz in der Zwischenzeit weniger dynamisch entwickelt, als wir es erwartet bzw. erhofft hatten. Darum halten wir die Beiträge dieses Bandes auch in der vorliegenden Form grundsätzlich noch für aussagekräftig. Aus diesem Grund wählen wir eine „Zwischenlösung" und machen die Texte allgemein zugänglich. Die vorliegenden Texte sind seitenidentisch mit denen der ersten Auflage.

Da diese Fassung mit der Schrift UWR-GaramondNo8, die Verlagsfassung aber mit Garamond gesetzt ist, kommt es in manchen Absätzen zu leichten Verschiebungen.

Wir danken allen Beitragenden für ihre Zustimmung zu dieser Form der Veröffentlichung.

Frankfurt am Main, Landau in der Pfalz, Januar 2010

Helma Lutz und Norbert Wenning 


\title{
Egalitäre Differenz in der Bildung
}

\author{
Annedore Prengel
}

Der folgende Beitrag setzt sich mit einem bestimmten Verständnis von Differenz auseinander: Die begriffliche Verbindung egalitäre Differenz eröffnet eine Perspektive, in der nach Verschiedenheit und nach Gleichberechtigung von Menschen gefragt wird. Egalität und Differenz werden nicht als gegensätzlich, sondern als einander wechselseitig bedingend verstanden. Egalität und Differenz sind dabei grundlegende Kategorien, um zu demokratischen Entwürfen von menschlichen Verhältnissen (Walzer 1992, Young 1997) zu finden. Keine der beiden Dimensionen ist in diesem $\mathrm{Zu}$ sammenhang verzichtbar, denn Gleichheit ohne Differenz würde undemokratische Gleichschaltung und Differenz ohne Gleichheit undemokratische Hierarchie hervorbringen.

Egalitär verstandene Differenz entspricht einer zugleich politischen, persönlichen und theoretischen Option: Im Gedanken der egalitären Differenz kommt der Wunsch zum Ausdruck, auf vielfältige Weisen leben zu können. Aus dem existentiellen Interesse, dass vielfältige Lebensweisen möglich sein mögen, geht auf theoretischer Ebene der Entwurf der egalitären Differenz hervor. Vielfalt des Lebens erscheint wertvoll, weil die Unterdrückung und Hierarchisierung von Lebensäußerungen als Verlust, Einschränkung, Störung oder gar als Zerstörung des Reichtums an Lebensmöglichkeiten, als „tort“ (Lyotard 1987), erfahren wird. Das Motiv, so Leid zu vermindern und Glück zu ermöglichen, wirkt sich auf die Art der Analyse und die Gestaltung von Bildungsprozessen aus: Egalitäre Differenz kann zu einem grundlegenden Erkenntnis- und Handlungsmotiv in schulischer und außerschulischer Bildungsforschung, -politik und -praxis werden und sich auf einzelne Menschen, Angehörige verschiedener Geschlechter, verschiedener Kulturen und Subkulturen, Menschen mit und ohne Behinderung sowie weitere Gruppierungen und die Beziehungen zwischen ihnen beziehen (Prengel 1993). Auch für wissenschaftliche Auseinandersetzungen eröffnet die Option der egalitären Differenz neue erkenntnissteigernde Möglichkeiten, da sie dazu anregt, sowohl nach Irrtümern und Grenzen als 
auch nach weiterführenden Einsichten und Potentialen eines jeden der antagonistischen Ansätze zu fragen (Blanck 1998, Zima 1999, Prengel 2000).

Einige aktuelle Interpretationen des Bildungsbegriffs orientieren sich an nichthierarchischen egalitären Vorstellungen von Differenz. Sie diskutieren das plurale Miteinander der Verschiedenen auf der Basis gleicher Rechte und wechselseitiger Anerkennung. Diese Bildungskonzepte sind verbunden mit einer essentialismuskritischen Offenheit für stets Neues hervorbringende Veränderungen in der Zeit, sei es als zu vermittelnde Bildungsinhalte, sei es als orientierendes Prinzip der Gestaltung von Bildungsprozessen in schulischen und außerschulischen Arbeitsfeldern oder sei es als Ziel von Bildungspolitik (vgl. z. B. Hörster 1995, Koch u. a. 1997, Marotzki 1988, Klafki 1994, Heyting/Tenorth 1994, Gogolin/Krüger-Potratz/ Meyer 1998, Hinz 1993, Flitner 1985, Benner/Tenorth 1996). In diesen Debatten geht es grundsätzlich darum, Ziele von Bildung und die ihnen innewohnenden Implikationen über menschliche Beziehungen zu klären. Sie formulieren und begründen auf ganz verschiedenen Wegen Konzepte egalitärer Differenz, die Kriterien zur Kritik undemokratischer Verhältnisse in pädagogischen Arbeitsfeldern zur Verfügung stellen. Die Ansätze, die sich mit der Bedeutung von Differenz für demokratische Bildungstheorie auseinandersetzen, beruhen allerdings ihrerseits auf in Praxissituationen von Pädagoginnen und Pädagogen und ihren Adressaten erarbeiteten Modellen des Miteinanders der Verschiedenen (vgl. Abschnitt 4).

Im Gedanken der egalitären Differenz kommt Kritik an hierarchischen Strukturen auf der gesellschaftlichen wie auf der persönlichen Ebene zum Ausdruck. Der antihierarchische Impuls korrespondiert mit den Qualifikations- und Sozialisationsfunktionen der Bildungsinstitutionen, da er Selbständigkeit im Umgang mit Wissen, eigenverantwortliches Handeln, Innovationsfähigkeit, Bewusstheit innerpsychischer Ambivalenzen sowie intersubjektive und interkulturelle Anerkennung anstrebt. Er kollidiert mit der Selektionsfunktion der Bildungsinstitutionen, die an die Stelle der historisch überholten geburtsständischen Hierarchiebildung im Zuge der Demokratisierung eine leistungsbegründete Hierarchiebildung setzen (von Friedeburg 1997, Luhmann 1990). Dieser Widerspruch durchzieht alle an egalitärer Differenz orientierten Bildungskonzepte; er muss transparent gemacht und umfassend untersucht werden, wenn diese Ansätze nicht unglaubwürdig werden wollen (Treptow/Hörster 1999, Prengel 1999, Hinz 1993, 1998, vgl. auch die Abschnitte 2 und 3). Das auf der Mikroebene in Wünschen und in alltäglich gelebten Praxen (vgl. Deppe-Wolfinger u. a. 1993) zum Ausdruck kommende Motiv der egalitären Differenz ist auf der gesellschaftlichen Makroebene zugleich in strukturellen Entwicklungen begründet: In Phänomenen wie Freiheit und eigener Lebensgestaltung zeigt sich ein „gesamtgesellschaftliches und nur begrenzt beeinflussbares Strukturprinzip der zweiten Moderne“ (Beck 1997, S. 22). Was auf der Mikro- 
ebene als Freiheit gelebt wird, ja gelebt werden muss, wird bei Analysen auf der Makroebene als Strukturprozess sichtbar. Dabei lassen sich beide Ebenen nicht ineinander überführen, weil Mikro- und Makroebene so unterschiedlich beschaffen sind, dass unterschiedliche theoretische Analyseinstrumente und unterschiedliche Forschungszugänge unerlässlich sind (Welz 1974).

Aus der Wertschätzung von Differenz und Egalität im Hinblick auf soziale Verhältnisse geht eine Denkweise hervor, die sich in Erkenntnisprozessen verschiedenen Facetten und widersprüchlichen Zusammenhängen widmet. Je mehr das Denken sich mehrperspektivische Zugänge (Prengel 1997) eröffnet, desto weiter entfernt es sich von monistischen Problemlösungen und schwarzweiß malenden Rechthabereien und desto mehr kann es sich den Ambivalenzen und dem pluralen Facettenreichtum von Untersuchungsgegenständen einschließlich der Widersprüche in den eigenen Entwürfen annähern. Egalitäre Differenz lässt sich darum nur in komplexen Denkfiguren erschließen. Einige für ihr Verständnis grundlegende Überlegungen möchte ich in diesem Beitrag zur Diskussion stellen. Er fasst in vier Punkten kurz einige Ergebnisse meiner theoretischen und empirischen Studien, die in zwanzig Jahren Auseinandersetzung mit der Frage der Verschiedenheit in der Bildung entstanden sind, zusammen: Teil 1 erinnert an die Notwendigkeit, bei der Rede über Differenz und Gleichheit ein „Tertium comparationis“ zu benennen. Teil 2 stellt Möglichkeiten und Grenzen der Vielfalt im historisch-gesellschaftlichen Kontext dar. Anschließend wird das Prinzip der egalitären Differenz als Analyseperspektive (Teil 3) und als Handlungsperspektive (Teil 4) erläutert.

\section{1 „Tertium comparationis" - der Zusammenhang von Gleichbeit und Differenz}

Über Gleichheit und Verschiedenheit zwischen Menschen kann man nur sinnvoll reden, wenn man ein sogenanntes „Tertium comparationis“ heranzieht, wenn man also sagt, in welcher Hinsicht jeweils von Gleichheit oder Differenz gesprochen werden soll (Radbruch 1950, Dann 1975, 1980, Gerhard 1990, Herberger/Neumann/Rüssmann 1992). Versäumte man, den Aspekt, der bei der Rede über Gleichheit oder Differenz angesprochen werden soll, zu präzisieren, würde man implizit pauschal so etwas wie absolute Identität oder absolute Differenz zwischen Menschen entwerfen, was unsinnig wäre. Gingen wir davon aus, dass wir uns uneingeschränkt gleichen, wie ein Ei dem anderen, wäre es sinnlos, Aussagen über Gleichheit zu machen. Dabei wird auch deutlich, wie fragwürdig es ist, pauschal Identität innerhalb einer Biographie anzunehmen, denn im Laufe der Zeit bleiben wir in vielerlei Hinsicht nicht dieselben, sogar die Körperzellen er- 
neuern sich, wie Wilhelm Windelband schon 1910 in seinem gleichheitstheoretischen Artikel zeigte (vgl. auch Klika 2000). Wenn man von Gleichheit redet, muss man also Differenz voraussetzen! Umgekehrt gilt ebenso: auch wenn man von Differenz redet, setzt man Gleichheit voraus, denn läge absolute Differenz ohne jeden Berührungspunkt, ohne jede Gemeinsamkeit vor, wäre es unmöglich, Aussagen, die für das voneinander Verschiedene gemeinsam gelten könnten, zu treffen. Beschreibe ich zum Beispiel Differenzen zwischen zwei Sprachen, setze ich schon voraus, dass sie sich im Hinblick darauf, dass sie beide Sprachen sind, gleichen; beschreibe ich Differenzen zwischen Kindern, habe ich schon vorausgesetzt, dass sie gemeinsam haben, Kinder zu sein; beschreibe ich Differenzen zwischen Kindern und Erwachsenen (siehe auch den Beitrag von Nemitz in diesem Band), habe ich schon vorausgesetzt, dass beide gemeinsam haben, Menschen zu sein oder einer Gruppierung von Menschen anzugehören usw. (zusammenfassend: Prengel 1993, S. 29-33).

Aus diesen Einsichten folgt, dass die Rede von Gleichheit und Differenz nur sinnvoll sein kann, wenn präzisiert wird, im Hinblick auf welchen Aspekt die jeweilige Aussage getroffen wird. Undifferenzierte Gleichheitsoder Unterschiedsbehauptungen halten kritischer Prüfung nicht stand und laufen Gefahr, von verborgenen politischen Intentionen bestimmt zu sein. So ist die Idee, mit der Differenz der Geschlechter müsse zwingend ihre Hierarchie verbunden sein, gerade in aktuellen feministischen Debatten populär (Gildemeister/Wetterer 1992). Anstelle solch pauschaler Schlüsse ist es notwendig, genau zu präzisieren, welche Differenz möglicherweise welche Hierarchisierungen fördern könnte und dabei zu berücksichtigen, dass ein (post-)moderner Differenzbegriff im Kern antihierarchisch und antiessentialistisch entworfen wurde und in scharfem Gegensatz zu essentialistischen Fixierungen aller Art steht.

\section{$2 \quad$ Vielfalt und Grenzen der Vielfalt}

Egalitäre Differenz ist die grundlegende - empirisch und theoretisch begründete - Idee der Pädagogik der Vielfalt, die ein nichthierarchisches, freiheitliches und entwicklungsoffenes Miteinander der Verschiedenen anstrebt. Diese Vielfalt in der Bildung steht im Konflikt mit der Selektionsfunktion des Bildungswesens, die die Einmündung in gesellschaftliche Statushierarchien aufgrund von linear-vergleichenden Leistungsbeurteilungen ermöglicht. Dieser Konflikt durchzieht alle Bildungsprozesse, auch wenn sie kindorientiert und offen für Heterogenität arbeiten. Darum muss er transparent gemacht und offen ausgetragen werden, wenn er sich nicht unter der Hand unerkannt auswirken soll (Prengel 1999). Aber darüber hinaus finden sich im Prinzip der egalitären Differenz selbst weitere Grenzen, die im Folgenden reflektiert werden. 
Menschen haben eine unermessliche Fülle an Lebensweisen hervorgebracht, zugleich sind wir endliche Wesen und können in den Grenzen unserer Lebensspanne nur begrenzt viele Lebensformen zu realisieren. Der politische Wille, Freiräume für vielfältige Lebensweisen offen zu halten, erübrigt darum nicht politische Entscheidungen, Freiheit für eine gewisse Zeit auf bestimmte Weise zu nutzen. Im Eröffnen von Freiräumen für die Überfülle des Vielfältigen zu verharren, käme aber dem Verzicht auf konkrete Lebensgestaltung gleich. Mit der Entscheidung so oder so zu leben, sei sie nun bewusst gefällt worden oder habe sie sich unbeabsichtigt ereignet, geht unvermeidlich eine mehr oder weniger starke, länger oder kürzer andauernde Beschränkung von Vielfalt einher. Pädagogik der Vielfalt kann sich deshalb um große Freiräume für ihre Adressaten in schulischen und außerschulischen Arbeitsfeldern bemühen, zugleich muss sie aber in jedem konkreten Arbeitsfeld, in jedem konkreten Bildungsvorhaben eine bestimmte Form annehmen und Bindungen eingehen, um überhaupt in pädagogisches Handeln übergehen zu können. Auch Theoriebildung und empirische Forschung sind ohne solche einschränkenden Entscheidungen für eine begrenzte Anzahl für das jeweilige Vorhaben ausgewählter theoretischer beziehungsweise methodischer Zugänge nicht möglich.

Dieser in aller Kürze skizzierte Zusammenhang ist Teil langfristiger gesellschaftlicher Entwicklungen von der Prämoderne über Moderne und Postmoderne zur Zweiten Moderne, die Wilhelm Schmid (1998) in seiner Philosophie der Lebenskunst untersuchte ${ }^{1}$. Während die Menschen in traditionalen gesellschaftlichen Kontexten an bestimmte Lebensformen gebunden sind, hat die Moderne diese Bindungen gelöst und Freiheit zur eigenen Lebensgestaltung ermöglicht. Den modernen Kampf um die Befreiung von alten bindenden Abhängigkeiten führt die Postmoderne weiter in einer Steigerung der Vielfalt ermöglichenden Freiheit. Aber diese immense Freiheit für unendlich viele Lebensmöglichkeiten existiert - analysiert man sie unabhängig von gesellschaftlich-strukturellen Einschränkungen, nach denen auch immer zu fragen ist - nur in der Zeitspanne vor der Wahl. In dem Maße, in dem ich meine Freiheit nutze und entscheide, wie ich leben will, gehe ich eine neue, jetzt selbst gewählte und auch in ihrer zeitlichen Dauer selbstbestimmte Bindung ein. Im Übergang von der potentiell unendlichen Vielfalt möglicher Differenzen zur Realisierung konkreter, be-

Dabei bezieht sich Prämoderne auf traditionale ständische Gesellschaften, in denen Lebenswege weitgehend vorbestimmt sind, Moderne auf die vor allem im Zuge der Industrialisierung ausgelöste Lösung aus solchen Bindungen und die langfristige gesellschaftliche Entwicklung hin zur Individualisierung, Postmoderne auf die Steigerung der Freiheit der Moderne in der Vervielfältigung denkbarer Lebensweisen und Zweite Moderne auf das Eingehen neuer, jetzt selbstgewählter und revidierbarer Bindungen. Die vier historischen Phasen Prämoderne, Moderne, Postmoderne, Zweite Moderne werden nicht als lineare, aufeinanderfolgende, sondern als auch teilweise gleichzeitig nebeneinander existierende Strömungen verstanden (Beck 1997, Schmid 1998). 
grenzter Differenz sieht Wilhelm Schmid die Weiterentwicklung von der Postmoderne hin zur Zweiten Moderne. Dabei dürfen Freiheit und Selbstbestimmung, wie oben ausgeführt, nicht mit Vorstellungen von der Willkür isolierter Einzelner verwechselt werden, sie sind vielmehr als im Sozialisationsprozess vermittelte strukturelle Erscheinungen aktueller Gesellschaften zu erklären.

Im Licht der Analysen von Schmid lässt sich der Gedanke der egalitären Differenz in der Bildung in seinen historischen Kontexten begreifen: In traditionalen Strukturen sollten Bildungseinrichtungen dazu dienen, den geburtsständischen Bestimmungen zur Geltung zu verhelfen. Kinder sollten zu dem Stand und Geschlecht erzogen werden, an den bzw. an das sie von Geburt an gebunden waren. Mit der Modernisierung des Bildungswesens wurde diese Art von Bindung zurückgedrängt. Bildung sollte nun die Freiheit eröffnen, sich aus den vorgegebenen Bindungen zu lösen, sich zu qualifizieren und kraft eigener Entscheidung und Leistungsfähigkeit Lebens- und Berufswege einzuschlagen. In den Bemühungen um Chancengleichheit durch kompensatorische Erziehung von soziokulturell und aufgrund ihrer Geschlechtszugehörigkeit benachteiligten Kindern sowie durch Erwachsenenbildung sollte das Leistungsprinzip des Bildungsparadigmas der Moderne noch einmal konsequenter eingelöst werden. Als postmodern lassen sich dann all jene Bildungskonzepte interpretieren, die, fußend auf dem Recht auf gleichen Zugang zur Bildung in der Moderne, der Wertschätzung heterogener Lebensweisen in und durch Bildungseinrichtungen zur Geltung verhelfen wollen und sich nicht auf Gleichstellung durch angleichende Assimilation reduzieren lassen.

Diese postmodernen Bildungskonzepte lösen die demokratischen Prinzipien der Moderne nicht ab, sondern enthalten sie im Kern, bauen auf ihnen auf und entwickeln sie weiter: Im Sinne von Freiheit sollen verschiedene Lebensweisen nicht unterdrückt werden, im Sinne von Gleichheit sollen verschiedene Lebensweisen nicht hierarchisiert werden und im Sinne von Brüderlichkeit sollen verschieden lebende Menschen sich wechselseitig existentiell anerkennen. Die faszinierenden postmodernen Freiheiten machen mannigfaltige und stets für Neues offene Bildungswege denkbar. Indem sie an konkreten Orten in konkreten Zeitspannen lebbar werden, beschränken sie sich, bei aller Offenheit und Flexibilität, unweigerlich auf bestimmte Formen und gehen ein in die auf Moderne und Postmoderne aufbauende Zweite Moderne. So konkretisiert sich Schulunterricht, der offen für die Vielfalt und Individualität kindlicher Persönlichkeiten sein will, in bestimmten ausgewählten pädagogischen Handlungsfiguren und entwickelt eigene, tendenziell dynamische und freiheitliche Rituale (Prengel 1999, Thurn/Tillmann 1997, Riegel 1994). Die prismatisch-poetische Gruppenarbeit von Alfred Drees (1997) ist ein Beispiel aus der Arbeit mit Erwachsenen für die Entwicklung eines bestimmten verbindlichen Rituals, das als 
Rahmen für größtmögliche Freiheiten hinsichtlich des persönlichen Ausdrucks dient.

\section{Egalitäre Differenz und Analyseperspektiven}

Wissenschaftliche Untersuchungen, auch in den Sozial- und Erziehungswissenschaften, werden nicht voraussetzungsfrei durchgeführt, denn die Art der Fragestellungen bedingt die Art der möglichen Erkenntnisse (vgl. zusammenfassend Prengel 1997, vgl. auch König 1989). Das Konstrukt der egalitären Differenz ist ein Erkenntnismotiv, das bestimmte Perspektiven auf eine Reihe von Ausschnitten der sozialen Welt eröffnet (vgl. Heinzel/ Prengel 1998) und damit bestimmte Erkenntnisse ermöglicht. Es beinhaltet die Suche nach Aussagen über egalitäre Aspekte mit Fragen nach Gemeinsamkeiten von Menschen und die Suche nach differenzierenden Aspekten mit Fragen nach Verschiedenheiten zwischen Menschen. Aus der Verbindung beider Fragestellungen, der Frage nach Gleichheit und der Frage nach Differenz geht das Bemühen hervor, sich der Heterogenität zwischen Menschen, also qualitativen Differenzen, die sich nicht in Hierarchie-, Komparativ-, Symmetrie- oder Analogiebildungen überführen lassen, anzunähern. Allerdings sind diese vergleichenden Denkformen auch für Analysen aus der Perspektive der egalitären Differenz nicht überflüssig, sie spielen sogar eine bedeutende Rolle in der Forschung, z. B., wenn es um Aussagen zur Verfügung über Ressourcen wie Geld, Raum und Zeit geht. Im Folgenden werde ich einige aus der Sicht des Konstrukts der egalitären Differenz wichtige Analyseperspektiven vorstellen.

Dabei ist es unerlässlich, sich klarzumachen, dass aus jeder von letztlich unendlich vielen verschiedenen möglichen Erkenntnisperspektiven jeweils nur begrenzte und unvollständige Aussagen möglich sind, die niemals verabsolutiert werden dürfen, etwa, indem sie einfach von einer Ebene auf eine andere übertragen werden (Welz 1974). Darüber hinaus ist an dieser Stelle auf die spannende Frage, die hier offen bleiben muss, zu verweisen, in welchem Maße wir durch unsere erkenntnisleitenden Konstrukte und die ihnen innewohnenden Interessen unsere Erkenntnisgegenstände prägen oder sogar selbst erst hervorbringen. Schließlich ist auch zu berücksichtigen, dass alle Aussagen aus den in den folgenden Abschnitten zu erörternden Perspektiven einerseits von erkennenden Personen versucht werden, die sich im Laufe des Erkenntnisprozesses selbst verändern und andererseits über zu erkennende Personen versucht werden, die sich ebenfalls im Laufe des Erkenntnisprozesses wandeln. Es ist also stets mit zu reflektieren, über welchen Weltausschnitt eines sozialen Lebenszusammenhangs gesprochen wird und für welche Zeitspanne die Aussage gelten soll.

Die universelle Perspektive ermöglicht Fragen nach Erkenntnissen über alle Menschen. Solche Fragen sind aus differenztheoretischer Sicht nicht 
etwa überflüssig und bringen nicht notwendigerweise „falsche Universalismen" ${ }^{\text {"2 }}$ hervor. Sie ermöglichen vielmehr Aufmerksamkeit für jene Aspekte menschlicher Existenz, die für die Existenz aller Menschen von Bedeutung sind. Dazu gehören unter anderem Natalität und Mortalität, Historizität und Sozialität sowie Generativität und Geschlechtlichkeit. Auf universeller Ebene ist die Option demokratischer Menschenrechte ${ }^{3}$ angesiedelt, mit der der Gedanke der egalitären Differenz auf das Engste verknüpft ist. Dabei ist die kritische Reflexion aller Aussagen auf dieser Ebene notwendig, wenn man nicht Gefahr laufen will, falschen Universalismen aufzusitzen. So gilt zum Beispiel universell, dass Menschen geboren werden, Lebensphasen durchlaufen und sterben, aber darüber, wie Geburt, Entwicklung und Tod (vgl. Wulf 1997) erlebt und gestaltet werden, lassen sich auf universeller Ebene keine Aussagen machen.

Die kollektive Perspektive ermöglicht Fragen nach Erkenntnissen über Gruppierungen von Menschen. Vor allem auf dieser Ebene spielen einige klassische differenztheoretische Diskurse eine Rolle, die nach der Konstitution des Verhältnisses von Normalität und Marginalität und der Bedeutung des „Anderen“, sei es als Weiblichkeit (Irigaray 1980), als Wahnsinn (Foucault 1971), als Behinderung (Moser 1995), als Kindheit (Aries 1978), als Minderheiten (Lyotard 1977) oder als „Fremdheit“ (Leiris 1985) konstruiert, fragen. Die genannten kollektiven Ausschnitte der sozialen Welt lassen sich in ein breites Spektrum vielfältiger weiterer Kollektive auffächern, zum Beispiel in Frauen/Männer aus verschiedenen Kulturen, Gesellschaftsschichten und Generationen, in Angehörige verschiedener Kulturen, die weiblich oder männlich, unterschiedlich alt und lesbisch, homosexuell oder heterosexuell, auf verschiedene Weise behindert beziehungsweise nichtbehindert sind oder in Kinder aus verschiedenen Subkulturen und mit verschiedenen Lernausgangslagen. Die Aufzählung ließe sich fortsetzen, sie zeigt, dass kollektive Zugehörigkeiten sich vielfältig durchkreuzen (Fraser 1996) und dass Menschen in der Regel gleichzeitig wie auch zeitlich aufeinanderfolgend unterschiedlichen Kollektiven angehören.

Mit Aussagen auf der kollektiven Ebene sind Probleme verbunden, die in den letzten Jahren zum Beispiel in der (Selbst-)Kritik von Frauenbewegung und Frauenforschung an der Kategorie Frau (Löw in diesem Band), in der Kritik der interkulturellen Erziehung (Diehm 2000) oder auch in der Kritik an sozial- und sonderpädagogischen Klassifikationen (Sander 1995, 1997, Kiesel/von Lüpke 1998) heftig diskutiert wurden. Einige Argumente

$2 \quad$ Falsche Universalismen sind Aussagen, die universelle Gültigkeit behaupten und dabei die auf der gewählten Aussageebene relevanten Differenzen zwischen Menschen verleugnen (Holland-Cunz 1999).

Universelle Bedeutung hat zum Beispiel das Verbot der Folter, da Menschen aus allen Kulturen, allen Altersgruppen und Geschlechtern traumatisierbar sind und Schmerzen empfinden (Dreitzel 1997) 
dieser Debatte lauten zum Beispiel, „Frau“ sei ein Konstrukt und keine Realität, Frauen unterschieden sich derart voneinander, dass keine allgemeinen Aussagen über Frauen möglich seien, mit der Verwendung der Kategorie „Frau“ reifiziere man das System der Zweigeschlechtlichkeit (Gildemeister/Wetterer 1992), die Theorie der Geschlechterdifferenz sei essentialistisch und schriebe damit weibliches Anderssein letztlich im Sinne ontologischer Wesensbestimmung der Frau auf neue Weise fest.

Entsprechend lautet die Kritik an weiteren im Bildungswesen relevanten Definitionen von Menschen mit Behinderungen, Lern- und Verhaltensstörungen und Krankheiten. Definitorische Aussagen über Gruppierungen aller Art und Differenzen zwischen ihnen sind also stets problematisch. Aber die Gefahr essentialistischer und etikettierender Zuschreibungen stellt sich nicht nur bei Aussagen auf der kollektiven Ebene ein, sondern ebenso bei Aussagen auf der universellen und der individuellen Ebene.

Die individuelle Perspektive ermöglicht Fragen nach Einzelpersonen; sie versucht, den Aspekt der Einzigartigkeit von Lebenssituationen und Biographien zu berücksichtigen. Für Bildungsprozesse ist diese Ebene besonders wichtig, weil sie von Anfang an, natürlich im sozioökonomischen Kontext der entstehenden bürgerlichen Gesellschaft, mit individuellen Lern- und Lebensläufen verbunden wurden und gegenwärtig noch stärker und auf neue Weise vom Anspruch der Individualisierung geprägt sind. Die individuelle Perspektive erfasst dabei nicht schon den kleinstmöglichen Ausschnitt, denn gerade aus differenztheoretischer Sicht stellt das Individuum keine Einheit mit abgeschlossener Identität dar. Darum lässt sich auch die individuelle Perspektive im Hinblick auf verschiedene Persönlichkeitsanteile, verschiedene Lebensabschnitte, verschiedene Beziehungsmöglichkeiten in verschiedenen sozialökologischen Kontexten wie Familie, Schule, Peer-group usw. auffächern. Identität kann so nur als Identität in Bewegung und in Fragmenten gedacht werden. Theoretische Analysen und empirische Forschungen können im Sinne eines dynamischen und für neu emergierende Facetten der Erkenntnisgegenstände offenen Differenzkonzepts fixierende Identifizierungen vermeiden - allerdings nur begrenzt: wenn überhaupt Aussagen über Einzelne wie über soziale Gruppierungen oder gar alle Menschen möglich sein sollen, müssen sie als Untersuchungseinheiten - zumindest provisorisch - definitorisch eingegrenzt werden.

Zusammenfassend lässt sich sagen: Immer, wenn wir Aussagen über soziale oder materielle Erkenntnisgegenstände treffen, reden wir in Interpretationskonstrukten und verfestigen damit in unseren Vorstellungen von sozialen Lebenszusammenhängen das so konstruierte Phänomen. An dieser Stelle wird deutlich, dass theoretische und empirische Kategorienbildung mit grundsätzlichen erkenntnistheoretischen Problemen verbunden ist. Diese wurden aus differenztheoretischer Sicht radikal aufgedeckt und im Zuge der Entwicklung der Denkfigur der egalitären Differenz in der Bil- 
dung von Anfang an reflektiert (Prengel 1984, 1993, vgl. auch Adorno 1980 und Habermas 1994). Michael Wimmer und Alfred Schäfer haben in ihren Überlegungen zu Repräsentation und Identifikation die hier zur Diskussion stehenden erkenntnistheoretischen Zusammenhänge präzisiert:

„Die Wirklichkeit ,auf den Begriff zu bringen' bedeutet immer schon das dem Begriff Fremde, Inkompatible als Nicht-Identisches zu negieren, womit andererseits die Repräsentationsfunktion des Begriffs fragwürdig wird. Wie ein begriffliches Denken möglich sein kann, das diese Differenz von bestimmender Identifikation und (immer auch) verfehlender Repräsentation zum Ausdruck zu bringen vermag, stellt eines der zentralen Probleme der Erkenntnistheorie und damit auch jeder Form der Kritik dar" (Wimmer) Schäfer 1999, S. 12 f.).

\section{$4 \quad$ Egalitäre Differenz und Handlungsperspektiven}

An zahlreichen Orten im Bildungswesen entwickelten sich Vorhaben, die gegen den Mainstream der Herstellung von Leistungshierarchien - der Auseinandersetzung mit Differenzen Raum geben, darin eine Chance für zukunftsweisende Innovationen sehen und reiche konkrete Erfahrungen gesammelt haben. Dazu gehören z. B. gemeinsames Lernen behinderter und nichtbehinderter Kinder (Dumke/Schäfer 1993, Döpp u. a. 1995), altersgemischtes Lernen in kleinen, aber auch in einigen großen Grundschulen (Knauf 1995), innere Differenzierung und Individualisierung im Unterricht von Reformschulen und breit angelegte Vorhaben der Schulreform (FaustSiehl u. a. 1995) oder auch einzelne Klassen an Regelschulen (Prengel 1999). Die hier seit Jahren untersuchten alltäglichen Erfahrungen regten zu theoretischen Reflexionen wie den Folgenden an:

Egalitäre Differenz als Handlungsmotiv von Bildung beruht auf dem Ziel des freiheitlichen, gleichberechtigten Zusammenlebens verschiedener Menschen. In Erziehungs- und Bildungssituationen sind mit dieser Zielsetzung, wie in anderen gesellschaftlichen Handlungsfeldern, stets mehrperspektivische Prozesse verbunden, die sich nicht auf eindimensional zielgerichtetes Handeln zuspitzen lassen, sondern das Wechseln zwischen verschiedenen Perspektiven favorisieren. Pädagogisches Handeln bedeutet hier u. a. das Ausbalancieren verschiedener Aspekte: Offenheit für die Lebensund Lernweisen der kindlichen, jugendlichen oder erwachsenen Adressaten, Offenheit für ihre Verschiedenheit, Bemühen um ihren gleichberechtigten Zugang zu Bildungseinrichtungen, Bemühen um ihre Qualifikation für gesellschaftliche (ökonomische, kulturelle) Teilhabe, Präsentation des Profils (der Inhalte, Ziele, Arbeitsweisen, Anforderungen) der Bildungseinrichtung, Klärung des Beziehungsangebots der Pädagoginnen und Pädagogen. Über diese prozessbezogenen Aspekte hinaus gehört zu an egalitärer Differenz orientierter Bildung das Miteinander der Verschiedenen auch als 
Bildungsinhalt, indem den Mitgliedern der heterogenen Lerngruppe angeboten und abverlangt wird, sich wechselseitig als Verschiedene kennen $\mathrm{zu}$ lernen und zu respektieren und erst auf dieser Grundlage auch zu streiten, $\mathrm{zu}$ konkurrieren und sich realistisch mit ihren persönlichen Stärken und Schwächen auseinander zu setzen (Deppe-Wolfinger u. a. 1993).

Damit geht eine mehrperspektivische Vorstellung von Leistung und Leistungsbewertung einher, die sowohl die intraindividuelle Entwicklung als auch die interindividuellen Interaktionen in heterogenen Gruppierungen, als auch in relativ leistungshomogenen Gruppierungen, als auch den interpersonellen Leistungsvergleich ermöglicht und keine dieser Erkenntnisperspektiven ausschließt. Landesweite, bundesweite oder gar weltweite Leistungsvergleiche werden in der Regel von Befürwortern heterogener Lerngruppen abgelehnt, aber ich finde, auch vergleichendes Wissen über Leistungsstände in Bildungseinrichtungen ist interessant und aufschlussreich, wenn es in den Möglichkeiten und Grenzen seiner Aussagekraft reflektiert und nicht zum alleinigen Maßstab für Erfolg oder Misserfolg gemacht wird. In der Schulpädagogik zeigen sich die verschiedenen Perspektiven auf Leistung besonders prägnant, sie erschließen aber auf andere Weise auch außerschulische Bildungsprozesse, zum Beispiel der Sozialpädagogik oder der Erwachsenenbildung, denn hier sind verschiedene Handlungsperspektiven ebenfalls unverzichtbar: individuelle Entwicklung und Kreativität fördern und zu Leistungsfähigkeit für eine selbständige Lebensbewältigung qualifizieren (Prengel 1999).

Wertschätzung von Differenz und Egalität bringt für das Verhältnis der erziehenden erwachsenen Generation zur zu erziehenden Kindergeneration besondere Implikationen mit sich. Grundlegend ist, für die eigenständig-kreativen Lebensäußerungen der Kinder Freiräume zu ermöglichen und diese Äußerungen zu achten und zu fördern. Damit kann die Wertschätzung für Entwürfe der jüngeren Generation insgesamt, für einzelne kinderkulturelle Gruppierungen sowie für jedes einzelne Kind einhergehen. Im Sinne der egalitären Differenz ist es selbstverständlich, dass Kindern die Fähigkeit zu Selbstachtung und Anerkennung der Anderen vermittelt werden soll, also, dass mit der Wertschätzung kindlicher Lebensäußerungen keineswegs verbunden ist, ausbeuterische oder übergriffige Handlungsweisen von Kindern gegenüber Kindern oder Erwachsenen zu dulden. Mit dem genannten Ziel ist also unverzichtbar die wechselseitige Verpflichtung auf Toleranz verknüpft. Aus einer Option für Differenz lässt sich nicht die Tolerierung der Zerstörung von Differenz ableiten.

Viel schwieriger ist es allerdings zu klären, wie Erwachsene sich zu solchen kreativen Lebensäußerungen von Kindern verhalten sollen, die kulturellen Konventionen zuwiderlaufen, ohne dass damit anderen Menschen Schaden zugefügt würde. Was ist zu tun, wenn Kinder oder Jugendliche es vermeiden, sich Kulturtechniken anzueignen? Eine dogmatische Interpreta- 
tion des Differenzprinzips, die die Maxime „es ist normal, verschieden zu sein" konsequent beherzigt, würde konsequente Offenheit auch gegenüber diesen Verhaltensmustern von Angehörigen der jüngeren Generation beinhalten, da sie keinem anderen schaden. An dieser Stelle kommt ein weiteres Argument ins Spiel: Im Generationenverhältnis hat die ältere Generation die Aufgabe, der jüngeren Generation alles das zu vermitteln, was sie zum Leben in einer bestimmten Gesellschaft und zur gleichberechtigten gesellschaftlichen Teilhabe so gut wie möglich befähigt. Damit werden Kinder mit Anforderungen konfrontiert, die Erwachsene als notwendig antizipieren. Die erziehenden Erwachsenen sind verantwortlich dafür, Kinder, so gut sie es vermögen, zum Überleben und darüber hinaus wohl auch zum Möglichst-Erfolgreich-Sein in der Wissens- und Informationsgesellschaft zu befähigen. Um beiden Prinzipien, der Anerkennung kreativer kindlicher Lebensäußerungen und der Qualifikation im Bereich der Kulturtechniken und des Wissens, gerecht zu werden, ist Erziehung vom Abwägen zwischen ihnen bestimmt. Weil unser Wissen über das, was Kinder brauchen, stets unvollkommen ist, können wir uns um angemessenes Ausbalancieren dabei nur bemühen und niemals sicher gehen. Darin liegt das Risiko jeder Erziehung - in der an egalitärer Differenz orientierten Gestaltung von Bildungsprozessen kommt es besonders deutlich zum Ausdruck.

\section{Literaturverzeichnis}

Adorno, Theodor W. 1980: Negative Dialektik. Frankfurt/M.: Suhrkamp

Aries, Philippe 1978: Geschichte der Kindheit. München: dtv

Beck, Ulrich (Hg.) 1997: Kinder der Freiheit. Frankfurt/M.: Suhrkamp

Benner, Dietrich/Tenorth, Heinz-Elmar 1996: Bildung zwischen Staat und Gesellschaft. In: Zeitschrift für Pädagogik. 42. Jg., 1996, Heft 1, S. 3-14

Blanck, Bettina 1998: Erwägen als philosophische Orientierung und Didaktik. In: Lohmann, Karl Reinhard/Schmidt, Thomas (Hg.) 1998: Akademische Philosophie zwischen Anspruch und Erwartung. Frankfurt/M.: Suhrkamp, S. 164-195

Dann, Otto 1975: Gleichheit. In: Brunner, Otto/Conze, Werner/Koselleck, Reinhart (Hg.) 1975: Geschichtliche Grundbegriffe. Historisches Lexikon zur politisch-sozialen Sprache in Deutschland. Stuttgart: Klett-Cotta, Bd. 2, S. 997-1046

Dann, Otto 1980: Gleichheit und Gleichberechtigung. Das Gleichheitspostulat in der alteuropäischen Tradition und in Deutschland bis zum ausgehenden 19. Jahrhundert. Berlin: Duncker u. Humblot

Deppe-Wolfinger, Helga/Prengel, Annedore/Reiser, Helmut 1993: Integrative Pädagogik in der Grundschule. Weinheim, München: Juventa

Diehm, Isabell 2000: Erziehung und Toleranz. Handlungstheoretische Implikationen Interkultureller Pädagogik. In: Zeitschrift für Pädagogik, 46. Jg., 2000, Heft 2, S. 251274

Döpp, Wiltrud/Hansen, Sylvie/Kleinespel, Karin 1995: Eine Schule für alle Kinder. Die Laborschule im Spiegel von Bildungsbiographien. Weinheim: Beltz

Drees, Alfred 1997: Innovative Wege in der Psychiatrie. Sozialstrategien und poetische Kommunikation. Giessen: Psychosozial-Verlag

Dreitzel, Hans-Peter 1997: Leid. In: Wulf 1997, S. 854-873

104 
Dumke, Dieter/Schäfer, Georg 1993: Entwicklung behinderter und nichtbehinderter Schüler in Integrationsklassen. Weinheim: Deutscher Studienverlag

Faust-Siehl, Gabriele/Schwabe, Anita/Stehr, Astrid 1995: Schulanfang ohne Auslese Rückschritt oder fällige Weiterentwicklung? In: Arbeitskreis aktuell, Mitteilungen des Grundschulverbandes. 1/1995, Nr. 49, S. 1-5

Flitner, Andreas 1985: Gerechtigkeit als Problem der Schule und als Thema der Bildungsreform. In: Zeitschrift für Pädagogik. 36. Jg., 1985, Heft 1, S. 1-26

Foucault, Michel 1971: Die Ordnung der Dinge. Frankfurt/M.: Suhrkamp

Fraser, Nancy 1996: Demokratie und Differenz. Eine feministische Perspektive der USamerikanischen Debatte. In: Frauenanstiftung e.V. 1996: Demokratie und Differenz. Feministische Bündnispolitik auf dem Weg zu einer Zivilgesellschaft. Hamburg: Frauenanstiftung

Friedeburg, Ludwig von 1997: Differenz und Integration im Bildungswesen der Moderne. In: Zeitschrift für Sozialisationsforschung und Erziehungssoziologie. 17. Jg., 1997, Heft 1, S. $42-55$

Gildemeister, Regine/Wetterer, Angelika 1992: Wie Geschlechter gemacht werden. Die soziale Konstruktion der Zweigeschlechtlichkeit und ihre Reifizierung in der Frauenforschung, in: Knapp, Gudrun Axeli/Wetterer, Angelika (Hg.) 1992: Traditionen Brüche. Entwicklungen feministischer Theorie. Freiburg: Kote, S. 201-254

Gogolin, Ingrid/Krüger-Potratz, Marianne/Meyer, Meinert A. (Hg.) 1998: Pluralität und Bildung. Opladen: Leske + Budrich

Habermas, Jürgen 1994: Das Falsche im Eigenen. Der Briefwechsel zwischen Theodor W. Adorno und Walter Benjamin. Ein Dokument kritischer Selbstvergewisserung aus der aufgeklärten deutschen Geistesrepublik. In: Die Zeit vom 23.9.1994, Nr. 39, S. $77 \mathrm{f}$.

Heinzel, Friederike/Prengel, Annedore 1998: Gemeinsam Leben und Lernen in der Grundschule. In: Horstkemper, Marianne/Zimmermann, Peter (Hg.) 1998: Dramatisierung und Individualisierung - Geschlechtstypische Sozialisation im Kindesalter. Opladen: Leske + Budrich, S. 83-107

Herberger, Maximilian/Neumann, Ulfried/Rüssmann, Helmut 1992: Generalisierung und Individualisierung im Rechtsdenken. Stuttgart: Franz Steiner

Heyting, Frieda/Tenorth, Heinz-Elmar (Hg.) 1994: Pädagogik und Pluralismus. Deutsche und niederländische Erfahrungen im Umgang mit Pluralität in Erziehung und Erziehungswissenschaft. Weinheim: Deutscher Studienverlag

Hinz, Andreas 1993: Heterogenität in der Schule. Hamburg: Curio

Hinz, Andreas 1998: Pädagogik der Vielfalt auch für Schulen in Armutsgebieten? Überlegungen zu einer theoretischen Weiterentwicklung. In: Hildeschmidt, A./Schnelle, I. (Hg.) 1998: Integrationspädagogik. Auf dem Weg zu einer Schule für alle. Weinheim, München: Juventa, S. 127-144

Holland-Cunz, Barbara 1999: Sieben Thesen zu einer feministischen Theorie der Demokratie. In: Abels, Gabriele/Sifft, Stefanie (Hg.) 1999: Demokratie als Projekt. Feministische Kritik an der Universalisierung einer Herrschaftsform. Frankfurt/M., New York: Campus

Hörster, Reinhard 1995: Pädagogisches Handeln. In: Krüger, Heinz-Hermann, Helsper, Werner (Hg.) 1995: Einführung in Grundbegriffe und Grundfragen der Erziehungswissenschaft. Opladen: Leske + Budrich, S. 35-42

Irigaray, Luce 1980: Speculum. Spiegel des anderen Geschlechts. Frankfurt/M.: Suhrkamp

Kiesel, Doron/Lüpke, Hans von (Hg.) 1998: Vom Wahn und vom Sinn. Krankheitskonzept in der multikulturellen Gesellschaft. Frankfurt/M.: Brandes und Aspel 
Klafki, Wolfgang 1994: Neue Studien zur Bildungstheorie und Didaktik. Zeitgemäße Allgemeinbildung und kritisch-konstruktive Didaktik. Weinheim, Basel: Beltz

Klika, Dorle 2000: Identität - ein überholtes Konzept? Kritische Anmerkungen zu aktuellen Diskursen außerhalb und innerhalb der Erziehungswissenschaft. In: Zeitschrift für Erziehungswissenschaft, 3. Jg., 2000, Heft 2, S. 285-304

Knauf, Anne (Hg.) 1995: Wege entstehen beim Gehen. Kleine Grundschulen entwickeln ein Konzept. Pädagogisches Institut Brandenburg. PLIB, Aufsatzsammlung. Ludwigsfelde

Koch, Lutz/Marotzki, Wilfried/Schäfer, Alfred (Hg.) 1997: Die Zukunft des Bildungsgedankens. Weinheim: Deutscher Studienverlag

König, Gert 1989: Perspektive, Perspektivismus, perspektivisch. In: Ritter, Joachim/ Gründer, Karlfried (Hg.) 1989: Historisches Wörterbuch der Philosophie. Basel: Schwabe, Sp. 363-375

Leiris, Michel 1985: Die eigene und die fremde Kultur. Frankfurt/M.: Suhrkamp

Luhmann, Niklas 1990: Die Homogenisierung des Anfangs: Zur Ausdifferenzierung des Schulsystems. In: Luhmann, Niklas/Schorr, Karl Eberhard (Hg.) 1990: Zwischen Anfang und Ende. Fragen an die Pädagogik. Frankfurt/M.: Suhrkamp, S. 73-111

Lyotard, Jean-Francois 1977: Das Patchwork der Minderheiten. Berlin: Merve

Lyotard, Jean-Francois 1987: Der Widerstreit. München: Wilhelm Fink

Marotzki, Winfried 1988: Bildung als Herstellung von Bestimmtheit und Ermöglichung von Unbestimmtheit. In: Hansmann, Otto/Marotzki, Winfried (Hg.) 1988: Diskurs Bildungstheorie I: Systematische Markierungen. Weinheim: Deutscher Studienverlag, S. 311-334

Moser, Vera 1995: Die Ordnung des Schicksals. Zur ideengeschichtlichen Tradition der Sonderpädagogik. Butzbach: AFRA

Prengel, Annedore 1984: Schulversagerinnen - Versuch über diskursive, sozialhistorische und pädagogische Ausgrenzungen des Weiblichen. Gießen: Focus

Prengel, Annedore 1993: Pädagogik der Vielfalt. Verschiedenheit und Gleichberechtigung in Interkultureller, Feministischer und Integrativer Pädagogik. Opladen: Leske + Budrich (2. Auflage 1995)

Prengel, Annedore 1997: Perspektivität anerkennen - Zur Bedeutung von Praxisforschung für Erziehung und Erziehungswissenschaft. In: Friebertshäuser, Barbara/ Prengel, Annedore (Hg.) 1997: Handbuch Qualitative Forschungsmethoden in der Erziehungswissenschaft. Weinheim, München: Juventa, S. 599-627

Prengel, Annedore 1999: Vielfalt durch gute Ordnung im Anfangsunterricht. Opladen: Leske + Budrich

Prengel, Annedore 2000: Interdiskursive Heterogenität - Zum Verhältnis von Integrationspädagogik, Sonderpädagogik und Allgemeiner Pädagogik. In: Friedrich, Albert/ Hinz, Andreas/Moser, Vera (Hg.) 2000: Perspektiven der Sonderpädagogik. Berlin: Luchterhand, S. 74-83

Radbruch, Gustav 1950: Rechtsphilosophie. Stuttgart: C. F. Müller

Riegel, Enja 1994: Rituale oder die Kultur des Zusammenlebens. In: Pädagogik, 46. Jg., 1994, Heft 1, S. 6-9

Sander, Alfred 1995: Zum Problem der Klassifikation in der Sonderschulpädagogik. Ein ökologischer Ansatz. In: Vierteljahresschrift für Heilpädagogik und ihre Nachbargebiete. 64. Jg., 1995, Heft 1, S. 15-31

Sander, Alfred 1997: Behinderungsgriffe und ihre Konsequenzen für die Integration. In: Eberwein, Hans (Hg.) 1997: Handbuch Integrationspädagogik. Weinheim: Beltz, S. 99-201 
Schmid, Wilhelm 1998: Philosophie der Lebenskunst. Eine Grundlegung. Frankfurt/M.: Suhrkamp

Thurn, Susanne/Tillmann, Klaus-Jürgen 1997: Unsere Schule ist ein Haus des Lernens. Das Beispiel Laborschule Bielefeld - Mit Kindern leben. Reinbek: Rowohlt

Treptow, Rainer/Hörster, Reinhardt (Hg.) 1999: Sozialpädagogische Integration. Entwicklungsperspektiven und Konfliktlinien. Weinheim, München: Juventa

Walzer, Michael 1992: Sphären der Gerechtigkeit. Ein Plädoyer für Pluralität und Gleichheit. Frankfurt/M.: Fischer

Welz, Rainer 1974: Probleme der Mehrebenenanalyse. In: Soziale Welt 2/1974, S. 169 185

Wimmer, Michael/ Schäfer, Alfred 1999: Einleitung. Zu einigen Implikationen der Krise des Repräsentationsgedankens. In: Schäfer, Alfred/Wimmer, Michael (Hg.) 1999: Identifikation und Repräsentation. Opladen: Leske + Budrich, S. 10-26

Windelband, Wilhelm 1910: Über Gleichheit und Identität. Heidelberg

Wulf, Christoph (Hg.) 1997: Vom Menschen. Handbuch Historische Anthropologie. Weinheim, Basel: Beltz

Young, Iris Marion 1997: Weder Gleichheit noch Vielfalt sind Patentrezepte. Der politische Prozeß in demokratischen Gesellschaften muß zwei Strategien gleichzeitig verfolgen. In: Frankfurter Rundschau vom 27.5.1997, S. 12

Zima, Peter V. 1999: Dialogische Theorie. Zum Problem der wissenschaftlichen Kommunikation in den Sozialwissenschaften. In: Ethik und Sozialwissenschaften. Streitforum für Erwägungskultur. 4/1999, Absatz 1-22 